\title{
INDECOMPOSABLE MATRICES DEFINING PLANE CUBICS
}

\author{
ANITA BUCKLEY
}

Abstract. In this article we find all (decomposable and indecomposable) $6 \times 6$ linear determinantal representations of Weierstrass cubics. As a corollary we verify the Kippenhahn conjecture for $\mathrm{M}_{6}$.

Mathematics subject classification (2010): 14M12, 14H52, 14H60.

Keywords and phrases: determinantal representations, plane cubics, vector bundles, Kippenhahn conjecture.

\section{REFERENCES}

[1] M. AтiYah, Vector bundles over an elliptic curve, Proc. London Math. Soc. (3), 7 (1957), 414-452.

[2] J. BACKelin, J. HerZoG, AND H. SANDERS, Matrix factorizations of homogeneous polynomials, Algebra Some Current Trends 1352, Lecture Notes in Mathematics, Springer (2006), 1-33.

[3] A. Beauville, Determinantal Hypersurfaces, Michigan Math. J. 48 (2000), 39-63.

[4] A. BeAUVILle, Vector bundles on curves and generalized theta functions: recent results and open problems, Complex Algebraic Geometry, MSRI Publications 28 (1995), 17-33.

[5] A. Buckley And T. KoŠIR, Plane Curves as Pfaffians, Annali Della Scuola Normale Superiore di Pisa-Classe di Scienze (2010).

[6] R. J. Cook And A. D. Thomas, Line bundles and homogeneous matrices, Quart. J. Math. Oxford Ser. (2) 30 (1979), 423-429.

[7] L. E. Dickson, Determination of All General Homogeneous Polynomials Expressible as Determinants with Linear Elements, Trans. Amer. Math. Soc. 22 (1921), 167-179.

[8] D. EISENBUD, Homological algebra on a complete intersection, with an application to group representations, Trans. Amer. Math. Soc. 260 (1980), 35-64.

[9] D. Eisenbud, The Geometry of Syzygies: A Second Course in Algebraic Geometry and Commutative Algebra, Graduate Texts in Mathematics, Springer (2005).

[10] H. Grassman, Die stereometrischen Gleichungen zweiten Grades, und die dadurch erzeugten Oberflächen, J. Reine Angew. Math. 49 (1855), 47-65.

[11] R. Kippenhahn, Über den Wertevorrat einer Matrix, Mathematische Nachrichten Volume 6, 3-4 (1951), 193-228.

[12] T. J. LAFFEY, A counterexample to kippenhahn's conjecture on hermitian pencils, Lin. Alg. Appl. 51 (1983), 179-182.

[13] P. LANCASTER AND L. Rodman, Canonical forms for symmetric / skew-symmetric real matrix pairs under strict equivalence and congruence, Linear Algebra Appl. 406, (2005), 1-76.

[14] Chi-Kwong Li and Ilya Spitkovsky, Equality of Higher Numerical Ranges of Matrices and a Conjecture of Kippenhahn on Hermitian Pencils, Lin. Alg. Appl. 58 (1998), 323-349.

[15] D. Mumford, Abelian varieties, Tata institute of fundamental research, Bombay, 1970.

[16] T. NetZer AND A. Thom, Polynomials with and without determinantal representations, Linear Algebra Appl. 437 (2012), 1579-1595.

[17] G. V. RAVINDRA AND AMIT TRIPATHI, Torsion points and matrices defining elliptic curves, International Journal of Algebra and Computation Vol. 24, 6, 2014.

[18] R. QuAReZ, Symmetric determinantal representation of polynomials, Linear Algebra Appl. 436 (2012), 3642-3660.

[19] H. SHAPIRO, A conjecture of Kippenhahn about the characteristic polynomial of a pencil generated by two Hermitian matrices, I and II, Lin. Alg. Appl. 43 (1982), 201-221 and 45 (1982), 97-108. 
[20] H. ShaPIRO, Hermitian pencils with a cubic minimal polynomial, Lin. Alg. Appl. 48 (1982), 81-103.

[21] F. SCHUR, Ueber die durch collineare Grundgebilde erzeugten Curven und Flächen, Mathematische Annalen, Vol. 181 (1881), 1-32.

[22] V. Vinnikov, Complete description of determinantal representations of smooth irreducible curves, Lin. Alg. Appl., 125 (1989), 103-140.

[23] V. VINNIKOV, Self-adjoint determinantal representations of real irreducible cubics, Operator Theory: Advances and Applications, 19 (1986), 415-442.

[24] V. VINNIKOV, LMI representations of convex semialgebraic sets and determinantal representations of algebraic hypersurfaces: Past, present, and future, Operator Theory: Advances and Applications, 222 (2012), 325-349.

[25] W. C. WATERHouse, The codimension of singular matrix pairs, Lin. Alg. Appl. 47 (1984), 227-245. 